\title{
Consumption of Hydrogen Water Reduces Paraquat-Induced Acute Lung Injury in Rats
}

\author{
Shulin Liu, ${ }^{1}$ Kan Liu, ${ }^{1}$ Qiang Sun, ${ }^{1}$ Wenwu Liu, ${ }^{1}$ Weigang Xu, ${ }^{1}$ \\ Petar Denoble, ${ }^{2}$ Hengyi Tao, ${ }^{1}$ and Xuejun Sun ${ }^{1}$ \\ ${ }^{1}$ Department of Diving Medicine, Faculty of Naval Medicine, Second Military Medical University, \\ 800 Xiangyin Road, Shanghai 200433, China \\ ${ }^{2}$ Divers Alert Network, Center for Hyperbaric Medicine and Environmental Physiology, Duke University, Durham, NC 27710, USA
}

Correspondence should be addressed to Hengyi Tao, taohengyi@hotmail.com and Xuejun Sun, sunxjk@hotmail.com

Received 3 September 2010; Accepted 9 January 2011

Academic Editor: Albert Zomaya

Copyright ( $) 2011$ Shulin Liu et al. This is an open access article distributed under the Creative Commons Attribution License, which permits unrestricted use, distribution, and reproduction in any medium, provided the original work is properly cited.

\begin{abstract}
Exposure to paraquat leads to acute lung injury and oxidative stress is widely accepted as a contributor to paraquat-induced acute lung injury. Recent studies have reported that consumption of water with dissolved molecular hydrogen to a saturated level (hydrogen water) prevents oxidative stress-induced diseases. Here, we investigated whether consumption of saturated hydrogen saline protects rats against paraquat-induced acute lung injury. Adult male Sprague-Dawley (SD) rats were randomly divided into four groups: Control group; hydrogen water-only group (HW group); paraquat-only group (PQ group); paraquat and hydrogen water group (PQ + HW group). The rats in control group and HW group drank pure water or hydrogen water; the rats in PQ group and PQ + HW group were intraperitonealy injected with paraquat $(35 \mathrm{mg} / \mathrm{kg})$ and then provided pure water or hydrogen water. Both biochemical and histological lung alterations were measured. The results showed that hydrogen water ameliorated these alterations, demonstrating that hydrogen water alleviated paraquat-induced acute lung injury possibly by inhibition of oxidative damage.
\end{abstract}

\section{Introduction}

Paraquat (1,1-dimethyl-4,4-bipyridilium dichloride, PQ) is a widely used contact and nonselective quaternary nitrogen herbicide. It was first introduced in agriculture in 1962 and has caused thousands of human deaths, either by accidental or voluntary ingestion. The toxicity of paraquat is based on its induction of redox cycling which leads to oxidative stressrelated cell death and inflammation. Because of selective accumulation in the lungs, it causes severe lung injury manifested by edema, hemorrhage, interstitial inflammation, and progressive fibrosis $[1,2]$. Many studies have suggested that the mechanisms of paraquat-induced injury are mainly associated with oxidative stress [3-5]. Paraquat-induced redox cycling can generate superoxide anions, $\mathrm{NO}$ and other free radicals, leading to oxidative damage $[2,6,7]$. Of all these free radicals, hydroxyl radicals and oxidant peroxynitrite might be the most toxic ones, as they react with biological macromolecules including DNA, proteins, and lipids, leading to DNA breakage, lipid peroxidation and protein inactivation. Hydroxyl radical is produced by superoxide anion and $\mathrm{H}_{2} \mathrm{O}_{2}$, respectively, through the HaberWeiss reaction and Fenton reaction $[8,9]$, while peroxynitrite is possibly induced by NO's rapid reaction with superoxide anion $[10,11]$.

Hydrogen, a colorless, tasteless, odorless, nonirritating, and highly flammable diatomic gas, was generally regarded as a physiologic inert gas in hyperbaric medicine. In 1975 and 2001, Dole et al. and Gharib et al., respectively, reported that hydrogen under a high pressure might be a therapeutic gas for cancer and parasite-induced liver inflammation by eliminating toxic $\operatorname{ROS}[12,13]$. In 2007, Ohsawa et al. found that $2 \%$ hydrogen inhalation exhibited antioxidant and antiapoptotic activities by selectively reducing hydroxyl radical and peroxynitrite [14]. The importance of hydrogen immediately drew widespread attention. Various ways to administrate hydrogen, such as $\mathrm{H}_{2}$ inhalation, oral administration of hydrogen water, intraperitoneal, and 
intravenous injection of hydrogen-saturated saline, have been proved to be effective for many ROS-induced diseases, including hepatic and cardiac hypoxia-ischemia injury, neonatal hypoxia-ischemia injury, human type II diabetes, nephrotoxicity induced by cisplatin and Parkinson's disease [15-20]. Particularly, lung injuries of various models can be also prevented by hydrogen [21-23]. Considering that oxidative stress, especially hydroxyl radical and peroxynitrite, contributes to paraquat-induced lung injury, we investigated the effect of oral administration of hydrogen on paraquatinduced inflammatory reaction, oxidative stress, and related damage of lungs, assessed by histological and biochemical parameters.

\section{Methods and Materials}

2.1. Animals. Forty adult male Sprague-Dawley rats weighing $250 \pm 10 \mathrm{~g}$ were used for this study. All experimental procedures were conducted in accordance with the Guiding Principle in the Care and Use of Animals approved by the Institutional Animal Care and Use Committee of Secondary Military Medical University, ROC.

2.2. Hydrogen Water Preparation. For the saturated hydrogen water preparation, purified $\mathrm{H}_{2}$ was dissolved into pure water for two hours under $0.6 \mathrm{MPa}$. The saturated hydrogen water was stored under atmospheric pressure at $4^{\circ} \mathrm{C}$ in an aluminum bag with no dead volume. Hydrogen water was freshly prepared every week, which maintained a continuous concentration. The hydrogen content was confirmed with a hydrogen electrode. Each day, hydrogen water from the aluminum bag was placed in a closed glass vessel, which ensured that the hydrogen concentration was greater than $0.4 \mathrm{mM}$ after one day. Hydrogen water degassed by gentle stirring was used for paraquat group; the complete removal of hydrogen gas was confirmed with a hydrogen electrode, similar to that of Ohsawa et al. [24].

2.3. Experimental Protocol. Paraquat was obtained from Sigma (St. Louis, MO, USA). Rats were randomly divided into four groups as follows: control group $(n=10)$; hydrogen water-only group (HW group, $n=10$ ); paraquat group (PQ group, $n=10$ ); paraquat and hydrogen water group (HW + PQ group, $n=10$ ). The rats in control group and HW group drank pure water or hydrogen water. The rats in PQ group and PQ + HW group were intraperitonealy injected with paraquat $(35 \mathrm{mg} / \mathrm{kg})$ and then administered pure water or hydrogen water ad libitum for $72 \mathrm{~h}$, respectively. To ensure the hydrogen concentration was greater than $0.4 \mathrm{mM}$, fresh hydrogen water was given every $12 \mathrm{~h}$. The concentration of $\mathrm{H}_{2}$ in blood after oral hydrogen water administration has been measured by Nagata et al., which was about $5 \mu \mathrm{M}$ [25]. At the end of $72 \mathrm{~h}$, animals were exsanguinated by abdominal aorta and followed by later procedures.

2.4. Measurement of Pleural Effusion and Lung Wet/Dry Weight Ratio. Immediately after abdominal aorta exsanguination, the pleural effusion was collected by inserting an 18-gauge needle and a $10-\mathrm{mL}$ syringe through the diaphragm and withdrawing all fluid present in the pleural cavity. Then lungs were excised en bloc and dissected away from the heart and thymus. The middle lobe of right lung was immediately weighed and then placed in a drying oven at $60^{\circ} \mathrm{C}$ for $96 \mathrm{~h}$ to stabilize dry weight. The ratio of wet/dry weight was used to quantify lung water content.

2.5. BALF Collection. BALF was performed on the left lung with $4 \mathrm{~mL}$ phosphate-balanced saline solution in 2.5$\mathrm{mL}$ aliquots after cannulation of the left trachea. The collected BALF was centrifuged at $1000 \mathrm{~g}$ for 10 minutes; the supernatant was collected and stored at $-20^{\circ} \mathrm{C}$ and $-80^{\circ} \mathrm{C}$ for later protein assays and LDH activity.

2.6. Total Cell Count, Total Protein, and Lactate Dehydrogenase (LDH) Activity in BALF. The total cell count was determined on a fresh fluid specimen using a hemocytometer. Total protein content in BALF was measured by the $\mathrm{BCA}$ protein assay reagents using BSA as a standard (Pierce, Rockford, IL, USA). The activity of LDH, an indicator for cellular oxidative damage, was measured at $490 \mathrm{~nm}$ using an LDH determination kit according to the manufacturer's instructions (Roche Molecular Biochemicals, Mannheim, Germany). LDH activity was expressed as U/L, using an LDH standard.

2.7. Lung Malondialdehyde (MDA) Measurement. Lung MDA levels were determined using an MDA Assay kit according to the operation manual (Beyotime, Haimen, China). Briefly, frozen lung tissues were homogenized. After centrifugation, free MDA in the supernatant was converted to a stable carbocyanin dye by the chemical reaction with N-methyl-2-phenylindole. Protein concentration was determined by the BCA Protein Assay (Pierce, Rockford, IL, USA) using BSA as a standard. MDA levels were normalized against protein $(\mathrm{pmol} / \mathrm{mg})$.

2.8. HßE and TUNEL Staining. After animals were exsanguinated by abdominal aorta, the lower lobe of right lungs was removed and then transferred to $4 \%$ formaldehyde for $48 \mathrm{~h}$. The lungs were paraffin embedded, and butterflyshaped sections of 5-mm thickness were cut and placed on glass microscope slides stained with hematoxylin and eosin (H\&E) for histopathological analysis. Apoptosis was detected by DNA strand breaks using terminal deoxynucleotidyl transferase-mediated biotinylated UTP nick end labeling (TUNEL) according to the procedure of the manufacturer (Bipec Biopharma, Massachusetts, MA). Average percentage of apoptotic cells was calculated in 10 randomly selected high power fields. Data were expressed as the ratio of TUNEL positive cells to total cells. Values are given as mean \pm SEM $(n=10)$.

2.9. Statistical Analysis. All values are presented as mean \pm SEM. Differences between groups were determined with one-way ANOVA followed by Student-Newman-Keuls test. A level of $P<.01$ was considered statistically significant. 


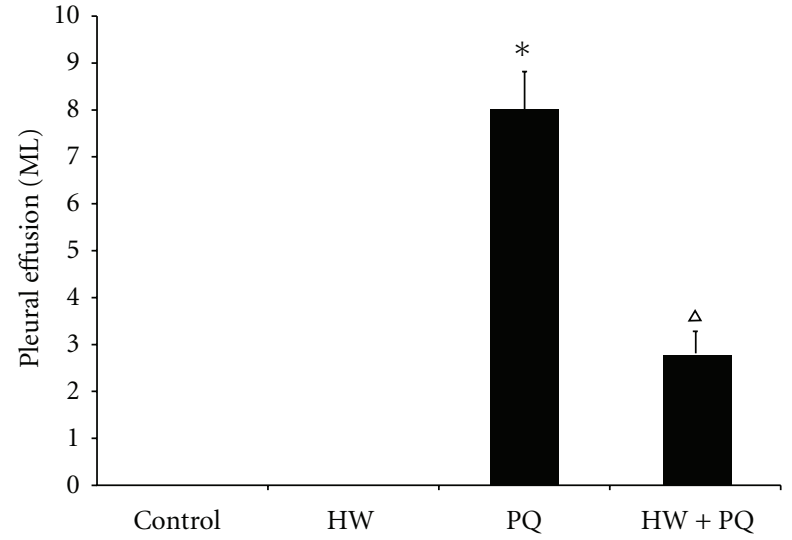

FIGURE 1: Effects of hydrogen water on pleural effusion. The rats in $\mathrm{PQ}$ group exhibited an increase in the volume of pleural effusion at the end of $72 \mathrm{~h}$, whereas in PQ + HW group this increase was significantly smaller $\left({ }^{*} P<.01\right.$ compared to control; ${ }^{\triangle} P<.01$ compared to PQ group).

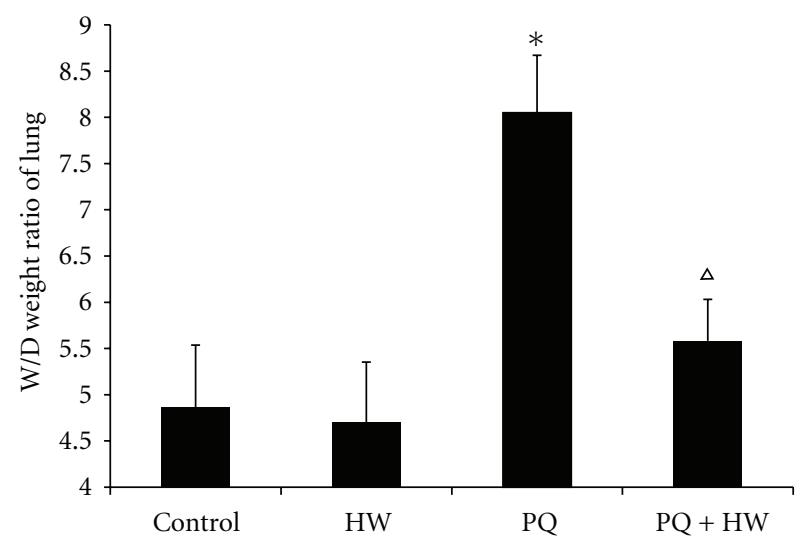

Figure 2: Effect of hydrogen water on lung edema. The lung wet/dry weight ratio of rats in PQ group was significantly higher than that of the control group $(P<.01)$. Compared to the $\mathrm{PQ}$ group, wet/dry weight ratio of rats in $\mathrm{PQ}+\mathrm{HW}$ group was significantly decreased $\left({ }^{*} P<.01\right.$ compared to control; ${ }^{\triangle} P<.01$ compared to PQ group).

\section{Results}

3.1. Effects of Hydrogen Water on Pleural Effusion. At 72 hours after paraquat injection both rats in PQ and PQ + HW groups had pleural effusions. However, the volume of effusions was significantly greater in PQ group $(8.0 \mathrm{~mL})$ than in PQ + HW group $(2.8 \mathrm{~mL}, P<.01)$ (Figure 1$)$.

\subsection{Effect of Hydrogen Water on Lung Edema. The wet/dry} weight ratio of rats was significantly higher in PQ group (8.5) than in the control group $(4.8, P<.01)$. In the PQ $+\mathrm{HW}$ group, the lung wet/dry weight ratio also increased (5.6) but it was significantly less than that in PQ group (Figure 2) $(P<.01)$.

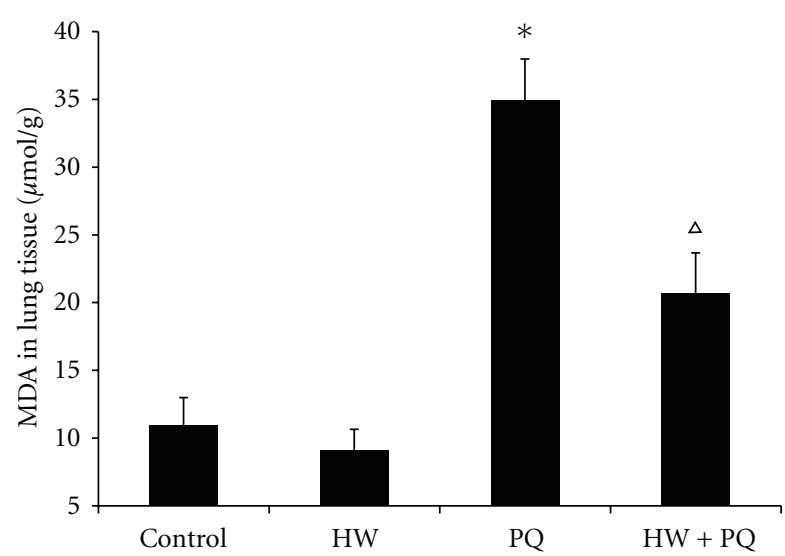

Figure 3: MDA in lung tissue. Our results demonstrated that paraquat increased lung MDA levels. In contrast, MDA levels were lesser in PQ + HW group $\left({ }^{*} P<.01\right.$ compared to control; ${ }^{\triangle} P<.01$ compared to PQ group).

TABLE 1: Effects of saturated hydrogen saline on LDH, total cells, and protein concentration in BALF. Rats exposed to paraquat exhibited significant increase in the $\mathrm{LDH}$ level in BAL fluid compared to rats in the control group, whereas a significant rather less of increase of LDH in BAL fluid was observed in PQ + HW group compared to those in PQ group. Total cells and protein concentration in the BAL fluid were determined as an indicator of lung hyperpermeability induced by paraquat. Our results showed that total cells and protein in BALF in PQ group were significantly increased compared to control group $(P<.01)$. In contrast, rats in $\mathrm{PQ}+\mathrm{HW}$ group demonstrated a significant lesser increase in total cells and protein $\left({ }^{*} P<.01\right.$ compared to control; ${ }^{\triangle} P<.01$ compared to PQ group).

\begin{tabular}{lccc}
\hline Groups & LDH $(\mathrm{U} / \mathrm{mL})$ & $\begin{array}{c}\text { Total cells } \\
\left(1 \times 10^{4} / \mathrm{mL}\right)\end{array}$ & $\begin{array}{c}\text { Protein } \\
\text { concentration } \\
(\mu \mathrm{g} / \mathrm{mL})\end{array}$ \\
\hline Control & $7.53 \pm 0.99$ & $15.65 \pm 1.29$ & $28.66 \pm 4.97$ \\
HW & $7.67 \pm 0.56$ & $15.96 \pm 1.98$ & $27.33 \pm 5.28$ \\
PQ & $70.38 \pm 2.14^{*}$ & $53.05 \pm 5.69^{*}$ & $80.33 \pm 3.88^{*}$ \\
PQ + HW $37.03 \pm 3.58^{* \Delta}$ & $30.13 \pm 6.60^{* \Delta}$ & $39.67 \pm 4.19^{* \Delta}$ \\
\hline
\end{tabular}

3.3. Effects of Saturated Hydrogen Saline on LDH, Total Cells, and Protein Concentration in BALF. Rats exposed to paraquat exhibited significant increase in the LDH level in BAL fluid compared to rats in the control groups $(P<$ $.01)$. However, the increase was significantly larger in $P Q$ $(70.38 \mathrm{U} / \mathrm{mL})$ than in $\mathrm{PQ}+\mathrm{HW}$ group $(30.13 \mathrm{U} / \mathrm{mL}, P<$ $.01)$. Similarly, total cells and protein in BALF were increased in both paraquat groups compared to control group $(P<$ $.01)$, but the increases were significantly larger in PQ than in $\mathrm{PQ}+\mathrm{HW}(P<.01)$ (Table 1$)$.

3.4. MDA in Lung Tissue. Lung MDA levels increased both in PQ group $(34.9 \mu \mathrm{mol} / \mathrm{g})$ and PQ + HW group $(20.7 \mu \mathrm{mol} / \mathrm{g})$ in comparison to control and HW groups $(10.9 \mu \mathrm{mol} / \mathrm{g}$, $9.1 \mu \mathrm{mol} / \mathrm{g}, P<.01)$. The increase, however, was significantly larger in PQ than in PQ + HW group (Figure 3 ). 


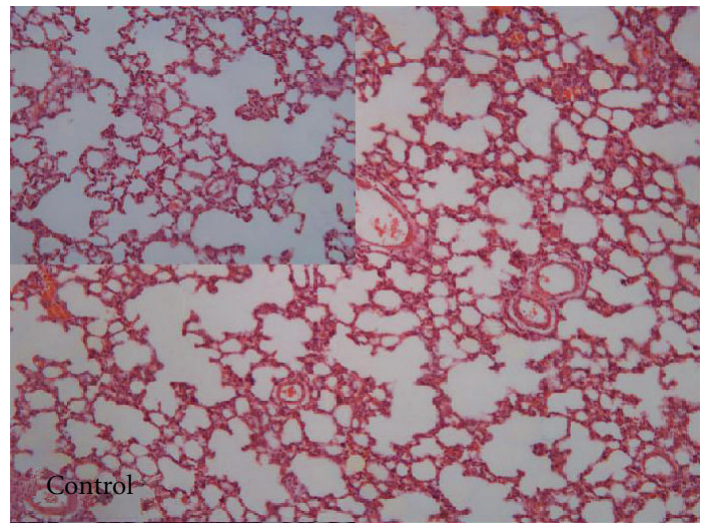

(a)

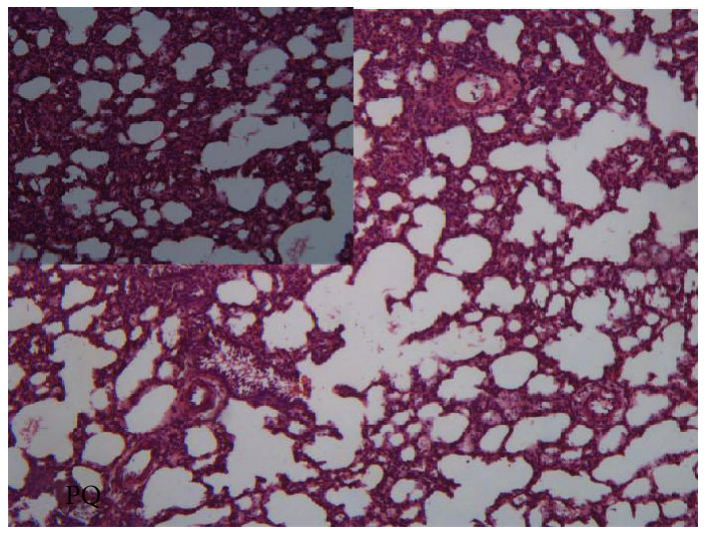

(c)

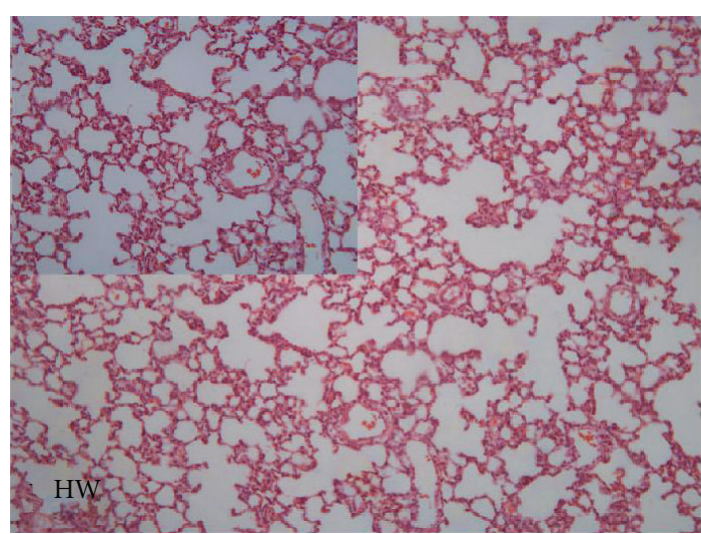

(b)

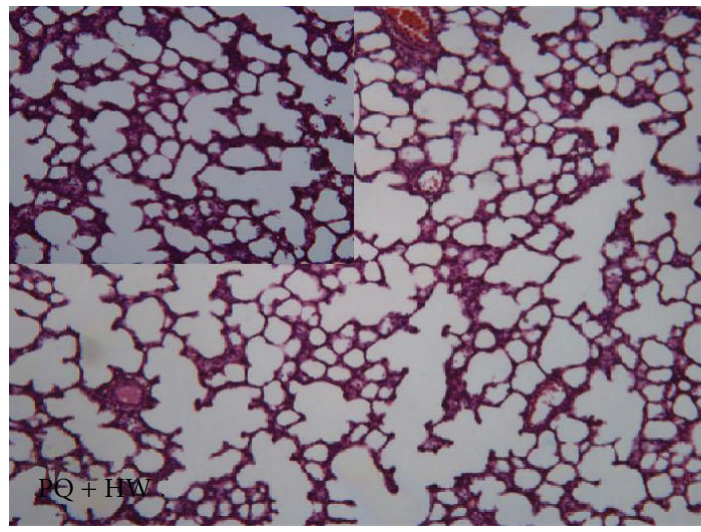

(d)

FIgURE 4: Histopathological examination by H\&E staining. Striking differences were observed in lung histology between the two experimental groups. Marked lung hemorrhage, edema, alveolar septal thickening, influx of inflammatory cells, and fibrin deposition were observed in rats injected with paraquat alone. In contrast, in PQ + HW group, the lung damage was alleviated, suggesting hydrogen water protected paraquat-induced acute lung injury.

3.5. Histopathological Examination by HeE Staining. Findings of histopathological analyses in lung slices prepared from rat in the three groups are shown in Figure 4. Striking differences were observed in lung histology between the two experimental groups. Marked lung hemorrhage, edema, alveolar septal thickening, influx of inflammatory cells, and fibrin deposition were observed in rats injected with paraquat alone. In contrast, in $\mathrm{PQ}+\mathrm{HW}$ group, similar changes were found but in lesser degree, suggesting an alleviation of the lung damage.

3.6. Effects of Saturated Hydrogen Saline on Cell Apoptosis. Rats in the PQ group exhibited a significant increase in the lung apoptotic index (14.3\%) compared with that in control group $(2.2 \%, P<.01)$. The apoptotic index was also increased in rats in PQ + HW group (6.9\%) but the increase was significantly less than in the PQ group (Figure 5).

\section{Discussion}

As there is no antidote for paraquat up to now, the treatment of paraquat poisoning consists mainly of attempts to decrease its absorption or enhance its elimination [26]. Since oxidative damage is known as the key issue in paraquat toxicity, several antioxidants have been studied to ameliorate paraquat-induced lung injuries. It is suggested that corticosteroids and cyclophosphamide may be effective in paraquat poisoning $[27,28]$. However, the effectiveness is still not agreed by others [29]. The protective effects on oxidative lung injury by other antioxidant were also studied, but the results turned out to be quite disappointing. GSH is ineffective in the protection of oxidant-induced lung injury as it is quickly cleared from the lung [30-32]. The effects of superoxide dismutase and catalase are limited due to their inability to cross biological membranes [26, 30, 3336]. Some other antioxidants, such as $\alpha$-tocopherol, provide limited protection because of their extreme insolubility [37]. Thus a quest for a suitable and effective treatment for paraquat-induced lung injury is still going on.

The present work was undertaken to determine the putative protective effect of hydrogen water on paraquatinduced lung injury. As hydrogen gas is explosive, the clinical application of it might be dangerous; we choose the oral administration of hydrogen water for treatment, 


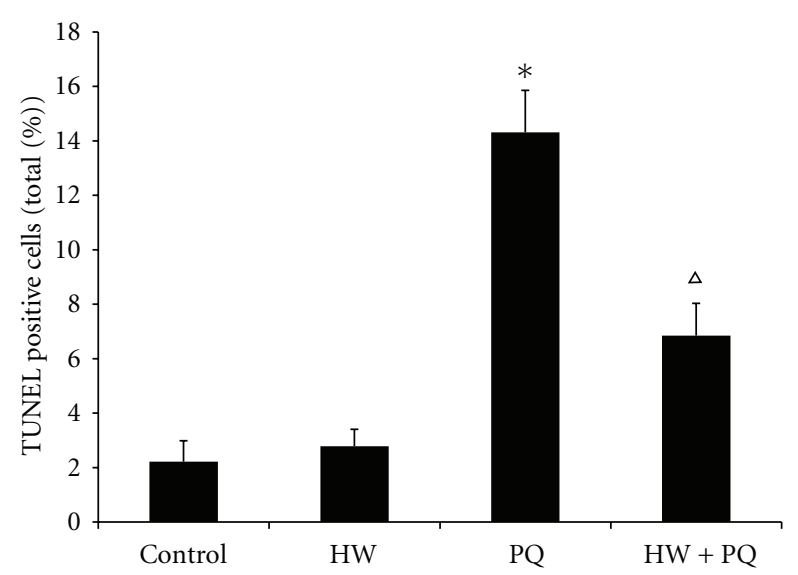

FIGURE 5: Effects of saturated hydrogen saline on cell apoptosis. Rats in the PQ group exhibit a significant induction in the lung apoptotic index compared with that in control group. Oppositely, the rats in PQ + HW group demonstrate a significant decrease in the lung apoptotic index compared with that in the PQ group, almost to the normal level $\left({ }^{*} P<.01\right.$ compared to control; ${ }^{\triangle} P<.01$ compared to PQ group).

the effectiveness of which has been validated previously $[24,25,38,39]$. Both the results of the biochemical assays and the histopathological findings of our study demonstrate that consumption of hydrogen water reduces the severity of paraquat-induced lung injury and oxidative stress in rats.

The rats in PQ + HW group exhibited significantly lesser degree of lung injury, as manifested by smaller pleural effusion volume, lesser protein accumulation and total cell count in the airways, and less morphologic abnormalities, when compared to PQ group. Lung W/d ratio in PQ + HW group was also significantly lower than that in the PQ group, demonstrating an ameliorated lung edema. The levels of $\mathrm{LDH}$ in BALF and MDA in lung tissue, known to be produced by peroxidation of cellular lipid and reliable indicators of oxidative damage $[15,22]$, were less in rats treated with hydrogen water, suggesting that hydrogen alleviates oxidative damage. The prevention of oxidative damage is the probable mechanism of the protection of lungs in rats exposed to paraquat and treated by hydrogen water.

Previous studies have shown that lung cell apoptosis is an important event in paraquat-induced lung injury [40]. The antiapoptotic effect of hydrogen was shown in neonatal hypoxia-ischemia rat model [17]. To determine whether hydrogen water exhibits the same inhibition of apoptosis in paraquat induced rat lungs injury, we examined lung cells apoptosis by TUNEL staining. We found a significant inhibition of cell apoptosis, consistent with previous findings.

Compared to traditional antioxidants, hydrogen, the newly explored antioxidant, offers a number of advantages. First, due to its small molecular weight, hydrogen can easily penetrate biomembranes and diffuse into the cytosol, mitochondria, and nucleus [15]. Second, as hydrogen selectively reacts with hydroxyl radical and peroxynitrite, other important ROS (e.g., $\mathrm{H}_{2} \mathrm{O}_{2}$ and $\mathrm{O}_{2}{ }^{-}$) involved in cell signaling are not decreased, so the metabolic oxidation-reduction reactions are not disturbed. Third, the tissue compatibility of hydrogen is greater than many other antioxidants. In lung, the application of $\mathrm{H}_{2}$ has unique benefits. On one hand, $\mathrm{H}_{2}$ has been applied as inhalation gas for many years in diving and has been proved to be safe to lungs [15]. On the other hand, as pulmonary blood flow is large and lung is the metabolism organ for $\mathrm{H}_{2}$, it can reach the lungs easily either after oral administration or injection of hydrogen-rich fluid. All of these properties of hydrogen make consumption of hydrogen water a promising treatment for a developing paraquat-induced lung injury.

\section{Conclusion}

To conclude, our study confirmed that hydrogen water reduces the paraquat-induced lung injury. Following these encouraging results, further studies should be performed to clarify these protective effects and to elucidate the exact mechanisms of this protection.

\section{Conflict of Interests}

The authors declare that there is no conflict of interests.

\section{Acknowledgments}

This study is supported by the National Natural Science Foundation of China (30971199). The authors thank Dr. Kang Zhiming and Dr. Li Runping in their laboratory for critically revising the paper.

\section{References}

[1] C. Bismuth, R. Garnier, F. J. Baud, J. Muszynski, and C. Keyes, "Paraquat poisoning: an overview of the current status," Drug Safety, vol. 5, no. 4, pp. 243-251, 1990.

[2] J. S. Bus and J. E. Gibson, "Paraquat: model for oxidantinitiated toxicity," Environmental Health Perspectives, vol. 55, pp. 37-46, 1984.

[3] M. A. Mussi and N. B. Calcaterra, "Paraquat-induced oxidative stress response during amphibian early embryonic development," Comparative Biochemistry and Physiology - C Toxicology and Pharmacology, vol. 151, no. 2, pp. 240-247, 2010.

[4] Z. Zhou, X. Chang, C. Shao, Q. Wu, Q. Wu, and M. Huang, "Pyrrolidine dithiocarbamate attenuates paraquat-induced lung injury in rats," Journal of Biomedicine and Biotechnology, vol. 2009, Article ID 619487, 2009.

[5] S. T. Y. Yeh, H. R. Guo, YU. S. Su et al., "Protective effects of $\mathrm{N}$-acetylcysteine treatment post acute paraquat intoxication in rats and in human lung epithelial cells," Toxicology, vol. 223, no. 3, pp. 181-190, 2006.

[6] A. P. Jaeger, J. Sauder, and J. Kopferschmitt, "Mechanisms of paraquat toxicity and herapeutic," in Paraquat Poisoning Mechanisms Prevention Treatment, C. Bismuth and A. H. Hall, Eds., pp. 141-159, Marcel Dekker, New York, NY, USA, 1995.

[7] Z. E. Suntres, "Role of antioxidants in paraquat toxicity," Toxicology, vol. 180, no. 1, pp. 65-77, 2002.

[8] B. Halliwell and J. M. C. Gutteridge, "Biologically relevant metal ion-dependent hydroxyl radical generation. An update," FEBS Letters, vol. 307, no. 1, pp. 108-112, 1992. 
[9] B. Halliwell and J. M. C. Gutteridge, "Oxygen free radicals and iron in relation to biology and medicine: some problems and concepts," Archives of Biochemistry and Biophysics, vol. 246, no. 2, pp. 501-514, 1986.

[10] H. I. Berisha, H. Pakbaz, A. Absood, and S. I. Said, "Nitric oxide as a mediator of oxidant lung injury due to paraquat," Proceedings of the National Academy of Sciences of the United States of America, vol. 91, no. 16, pp. 7445-7449, 1994.

[11] B. Nemery and R. J. van Klaveren, "NO wonder paraquat is toxic," Human \& Experimental Toxicology, vol. 14, no. 3, pp. 308-309, 1995.

[12] M. Dole, F. R. Wilson, and W. P. Fife, "Hyperbaric hydrogen therapy: a possible treatment for cancer," Science, vol. 190, no. 4210, pp. 152-154, 2007.

[13] B. Gharib, S. Hanna, O. M. S. Abdallahi, H. Lepidi, B. Gardette, and M. De Reggi, "Anti-inflammatory properties of molecular hydrogen: investigation on parasite-induced liver inflammation," Comptes Rendus de l'Academie des Sciences Serie III, vol. 324, no. 8, pp. 719-724, 2001.

[14] I. Ohsawa, M. Ishikawa, K. Takahashi et al., "Hydrogen acts as a therapeutic antioxidant by selectively reducing cytotoxic oxygen radicals," Nature Medicine, vol. 13, no. 6, pp. 688-694, 2007.

[15] K. I. Fukuda, S. Asoh, M. Ishikawa, Y. Yamamoto, I. Ohsawa, and S. Ohta, "Inhalation of hydrogen gas suppresses hepatic injury caused by ischemia/reperfusion through reducing oxidative stress," Biochemical and Biophysical Research Communications, vol. 361, no. 3, pp. 670-674, 2007.

[16] K. Hayashida, M. Sano, I. Ohsawa et al., "Inhalation of hydrogen gas reduces infarct size in the rat model of myocardial ischemia-reperfusion injury," Biochemical and Biophysical Research Communications, vol. 37, no. 1, pp. 330-335, 2008.

[17] J. Cai, Z. Kang, W. W. Liu et al., "Hydrogen therapy reduces apoptosis in neonatal hypoxia-ischemia rat model," Neuroscience Letters, vol. 441, no. 2, pp. 167-172, 2008.

[18] S. Kajiyama, G. Hasegawa, M. Asano et al., "Supplementation of hydrogen-rich water improves lipid and glucose metabolism in patients with type 2 diabetes or impaired glucose tolerance," Nutrition Research, vol. 28, no. 3, pp. 137143, 2008.

[19] N. Nakashima-Kamimura, T. Mori, I. Ohsawa, S. Asoh, and S. Ohta, "Molecular hydrogen alleviates nephrotoxicity induced by an anti-cancer drug cisplatin without compromising antitumor activity in mice," Cancer Chemotherapy and Pharmacology, vol. 64, no. 4, pp. 753-761, 2009.

[20] Y. Fu, M. Ito, Y. Fujita et al., "Molecular hydrogen is protective against 6-hydroxydopamine-induced nigrostriatal degeneration in a rat model of Parkinson's disease," Neuroscience Letters, vol. 453, no. 2, pp. 81-85, 2009.

[21] Y. F. Mao, X. F. Zheng, J. M. Cai et al., "Hydrogen-rich saline reduces lung injury induced by intestinal ischemia/reperfusion in rats," Biochemical and Biophysical Research Communications, vol. 381, no. 4, pp. 602-605, 2009.

[22] J. Zheng, K. Liu, Z. Kang et al., "Saturated hydrogen saline protects the lung against oxygen toxicity," Undersea and Hyperbaric Medicine, vol. 37, no. 3, pp. 185-192, 2010.

[23] C. S. Huang, A. Nakao, S. Lee et al., "Hydrogen gas inhalation attenuates ventilator-induced lung injury in mice," American Journal of Respiratory and Critical Care Medicine, vol. 181, A3751 pages, 2010.
[24] I. Ohsawa, K. Nishimaki, K. Yamagata, M. Ishikawa, and S. Ohta, "Consumption of hydrogen water prevents atherosclerosis in apolipoprotein E knockout mice," Biochemical and Biophysical Research Communications, vol. 377, no. 4, pp. 1195-1198, 2008.

[25] K. Nagata, N. Nakashima-Kamimura, T. Mikami, I. Ohsawa, and S. Ohta, "Consumption of molecular hydrogen prevents the stress-induced impairments in hippocampus-dependent learning tasks during chronic physical restraint in mice," Neuropsychopharmacology, vol. 34, no. 2, pp. 501-508, 2009.

[26] Z. E. Suntres, S. R. Hepworth, and P. N. Shek, "Protective effect of liposome-associated $\alpha$-tocopherol against paraquatinduced acute lung toxicity," Biochemical Pharmacology, vol. 44, no. 9, pp. 1811-1818, 1992.

[27] E. Addo, S. Ramdial, and T. Poon-King, "High dosage cyclophosphamide and dexamethasone treatment of paraquat poisoning with $75 \%$ survival," West Indian Medical Journal, vol. 33, no. 4, pp. 220-226, 1984.

[28] J. L. Lin, M. L. Leu, Y. C. Liu, and G. H. Chen, "A prospective clinical trial of pulse therapy with glucocorticoid and cyclophosphamide in moderate to severe paraquat-poisoned patients," American Journal of Respiratory and Critical Care Medicine, vol. 159, no. 2, pp. 357-360, 1999.

[29] N. A. Buckley, "Pulse corticosteroids and cyclophosphamide in paraquat poisoning," American Journal of Respiratory and Critical Care Medicine, vol. 163, no. 2, p. 585, 2001.

[30] M. Jurima-Romet, R. F. Barber, J. Demeester, and P. N. Shek, "Distribution studies of liposome-encapsulated glutathione administered to the lung," International Journal of Pharmaceutics, vol. 63, no. 3, pp. 227-235, 1990.

[31] R. N. Puri and A. Meister, "Transport of glutathione, as $\gamma$ glutamylcysteinylglycyl ester, into liver and kidney," Proceedings of the National Academy of Sciences of the United States of America, vol. 80, no. 17 I, pp. 5258-5260, 1983.

[32] L. J. Smith, J. Anderson, and M. Shamsuddin, "Glutathione localization and distribution after intratracheal instillation: implications for treatment," American Review of Respiratory Disease, vol. 145, no. 1, pp. 153-159, 1992.

[33] Z. E. Suntres and P. N. Shek, "Liposomal $\alpha$-tocopherol alleviates the progression of paraquat-induced lung damage," Journal of Drug Targeting, vol. 2, no. 6, pp. 493-500, 1995.

[34] M. J. Poznansky and R. L. Juliano, "Biological approaches to the controlled delivery of drugs: a critical review," Pharmacological Reviews, vol. 36, no. 4, pp. 277-336, 1984.

[35] J. F. Turrens, J. D. Crapo, and B. A. Freeman, "Protection against oxygen toxicity by intravenous injection of liposomeentrapped catalase and superoxide dismutase," Journal of Clinical Investigation, vol. 73, no. 1, pp. 87-95, 1984.

[36] R. V. Padmanabhan, R. Gudapaty, and I. E. Liener, "Protection against pulmonary oxygen toxicity in rats by the intratracheal administration of liposome-encapsulated superoxide dismutase or catalase," American Review of Respiratory Disease, vol. 132, no. 1, pp. 164-167, 1985.

[37] J. H. Senior, G. Gregoriadis, D. P.R. Muller, Y. V. Pathak, and N. McIntyre, "Liposomes facilitate uptake of lipid-soluble vitamins after oral delivery to normal and bile-duct obstructed rats," Biochemical Society Transactions, vol. 17, no. 1, pp. 121122, 1989.

[38] M. Kajiya, M. J. B. Silva, K. Sato, K. Ouhara, and T. Kawai, "Hydrogen mediates suppression of colon inflammation induced by dextran sodium sulfate," Biochemical and Biophysical Research Communications, vol. 386, no. 1, pp. 1115, 2009. 
[39] Y. Sato, S. Kajiyama, A. Amano et al., "Hydrogen-rich pure water prevents superoxide formation in brain slices of vitamin C-depleted SMP30/GNL knockout mice," Biochemical and Biophysical Research Communications, vol. 375, no. 3, pp. 346350, 2008.

[40] R. J. Dinis-Oliveira, C. Sousa, F. Remião et al., "Sodium salicylate prevents paraquat-induced apoptosis in the rat lung," Free Radical Biology and Medicine, vol. 43, no. 1, pp. 48-61, 1975. 


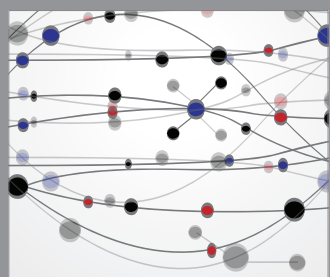

The Scientific World Journal
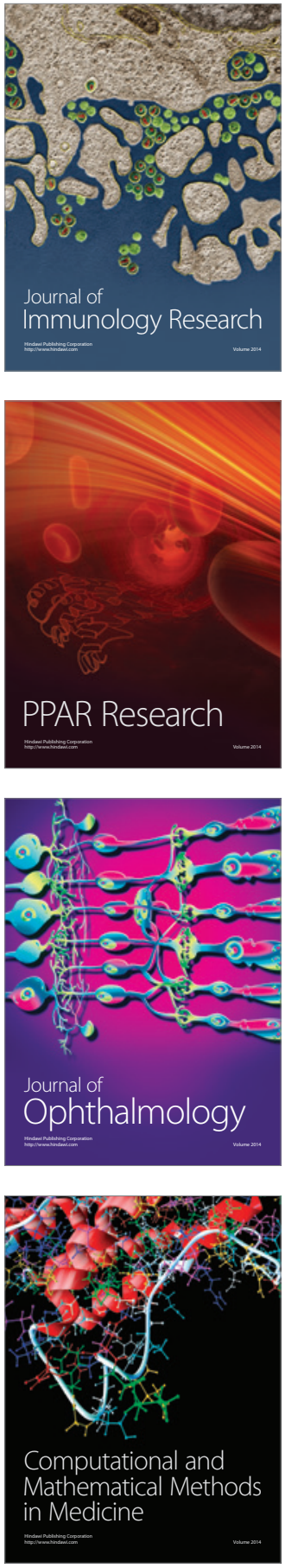

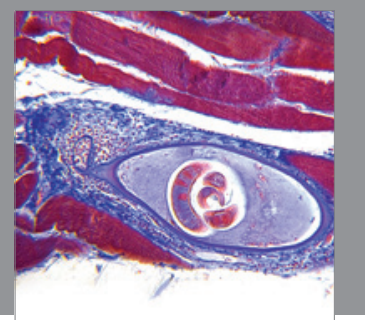

Gastroenterology

Research and Practice
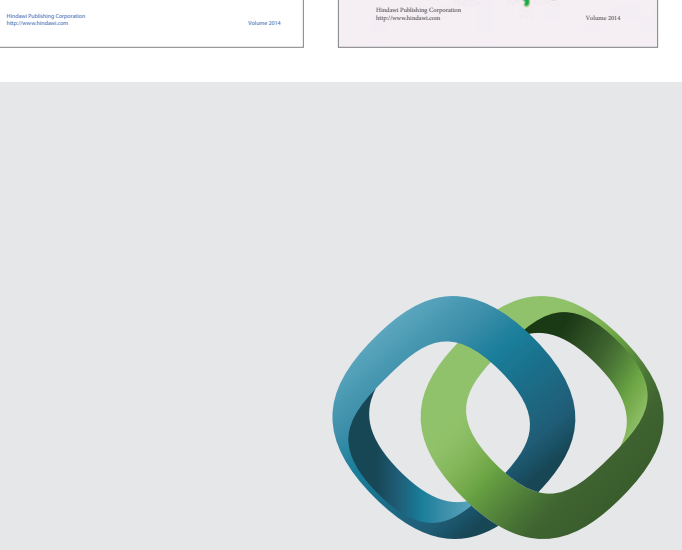

\section{Hindawi}

Submit your manuscripts at

http://www.hindawi.com
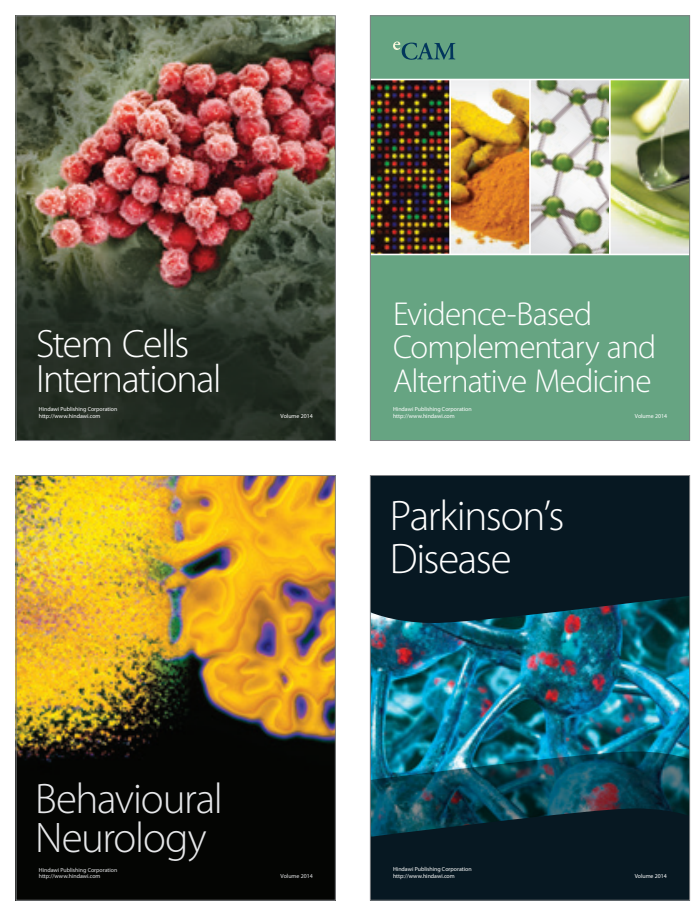

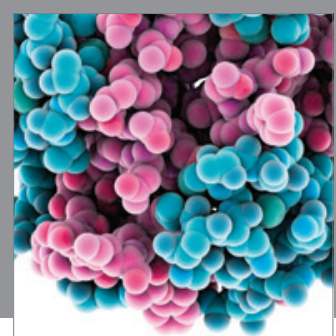

Journal of
Diabetes Research

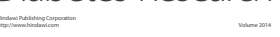

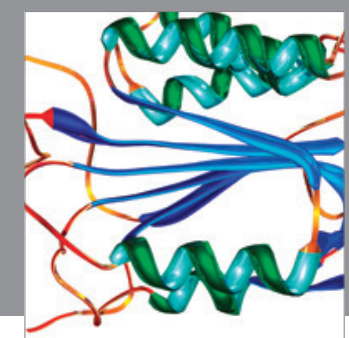

Disease Markers
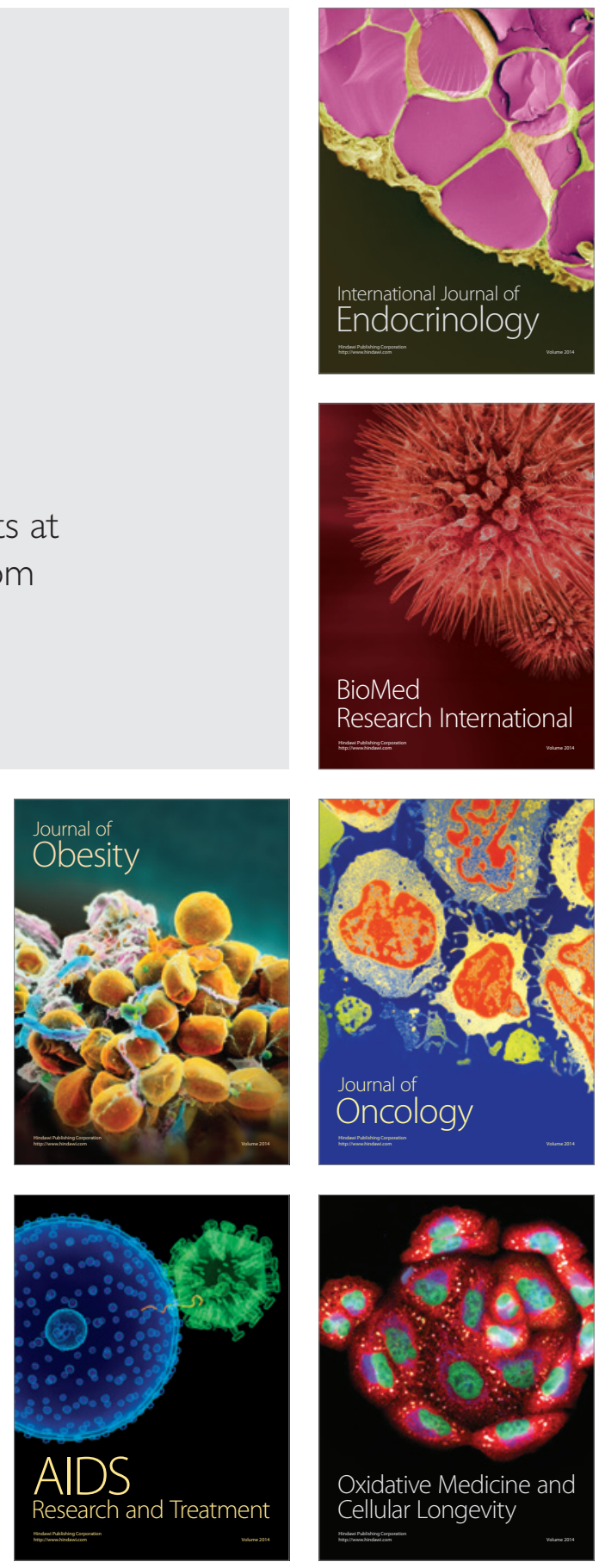